\title{
The Crystalline Enol of 1,3-Cyclohexanedione and Its Complex with Benzene: Vibrational Spectra, Simulation of Structure and Dynamics and Evidence for Cooperative Hydrogen Bonding
}

\author{
Bruce S. Hudson,* Dale A. Braden, ${ }^{\dagger}$ Damian G. Allis, Tim Jenkins, Sergey Baronov, \\ Chris Middleton," \\ Department of Chemistry, Syracuse University, Syracuse, New York 13244-4100; Schrödinger, Inc., \\ 1500 S. W. First Avenue, Suite 1180, Portland, Oregon 97201-5815; School of Chemical and Life Sciences, \\ University of Greenwich, Chatham Maritime Campus, Chatham, Kent ME4 4TB, United Kingdom; and \\ Department of Materials Science and Engineering, University of Maryland, \\ College Park, Maryland 20742-2115 and NIST Center for Neutron Research, \\ National Institute of Standards and Technology, Gaithersburg, Maryland 20899-8562
}

Received: March 30, 2004

\begin{abstract}
The inelastic incoherent neutron scattering spectra of 1,3-cyclohexanedione (CHD) in its crystalline enol form and its cyclamer complexes with benzene and benzene- $d_{6}$ are compared with each other, with IR and Raman spectra and with the results of calculations using density functional theory (DFT). The crystal packing of the CHD enol is a linear hydrogen-bonded chain with conjugated donor and acceptor groups analogous to that found in peptide hydrogen bonding. The benzene complex is a closed hexameric hydrogen-bonded cycle. A DFT treatment is applied to the full hexamer of the benzene complex. The CHD chain is treated as a series of finite linear clusters by DFT, while the infinite one-dimensional chain and the three-dimensional crystal are treated by periodic DFT. Comparison is made with both the observed crystal structures and the vibrational spectra. The very good to excellent agreement of the computed vibrational spectra with experiment demonstrates that the models and computational treatments used are reliable. The theoretical treatment of the linear chain clusters exhibits a continuous change in structure with increasing chain length, converging to values near the observed structure. Emphasis is placed on the cooperative nature of hydrogen bonding in CHD as revealed by these systematic trends. The ability of DFT methods to treat hydrogen bonding in solids appears to be roughly as accurate as the crystal structure determinations.
\end{abstract}

1,3-Cyclohexanedione (CHD) exists in its enol form in the solid state where it forms hydrogen-bonded chains with an antianti configuration having short $(2.56 \AA) \mathrm{O}-\mathrm{O}$ hydrogen bonding distances. ${ }^{1}$ This structure is of interest for several reasons including the ordered hydrogen bonding arrangement, an orderdisorder phase transition that involves hydrogen bond interchange, ${ }^{2-6}$ and indications derived from examination of crystal structures that CHD is an example of a resonance-assisted, cooperative hydrogen bonding network. ${ }^{7-13}$ This cooperativity arises from the fact that when the enol of CHD forms a hydrogen bond, this polarizes the molecule such that the formation of an additional hydrogen bond with another CHD is more favorable. This may be thought of as due to an enhanced contribution of the ionic resonance form. When crystallized from benzene, this material forms a unique cyclamer structure $\left(\mathrm{CHD}_{6} \mathrm{Bz}\right)$ in which six CHD molecules form a syn-anti hydrogen bonded ring surrounding a central benzene molecule. ${ }^{14}$

The related 1,3-cyclopentanedione $(\mathrm{CPD})^{15}$ forms an antianti linear chain structure very much like that of CHD. Methyl substitution of CPD or CHD can result in changes to the hydrogen-bonding pattern. 2-Methyl-CPD forms a linear chain

* Corresponding author. E-mail: bshudson@syr.edu.

$\dagger$ Schrödinger, Inc.

$\doteqdot$ Syracuse University. Current address: Moscow State University.

II Syracuse University. Current address: The Ohio State University.

$\S$ University of Greenwich, Chatham Maritime Campus.

\# University of Maryland.
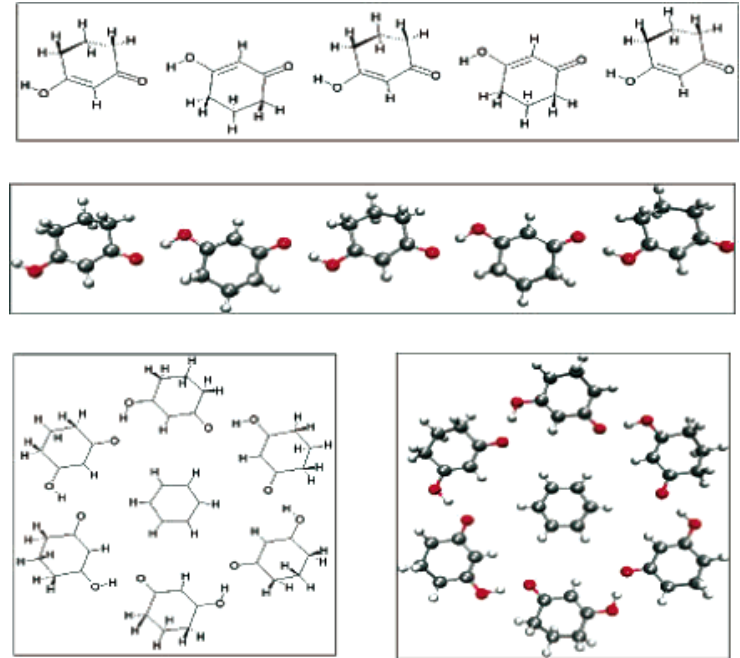

Figure 1. $\mathrm{CHD}$ chain and $\mathrm{CHD}_{6} \mathrm{Bz}$ cyclamer structures.

with an anti-syn arrangement. ${ }^{16}$ In this case the methyl-CHD molecules in a given linear chain all point in the same direction. This is in contrast to the anti-anti chain of CPD and CHD (Figure 1) where alternate molecules are oriented in opposite directions. The 2-methyl derivative of CHD has a structure similar to that of $\mathrm{CHD}^{17}$ but the 5,5-dimethyl derivative ("dimedone") forms a syn-syn helical structure with an $\mathrm{O}-\mathrm{O}$ 
hydrogen bond distance of $2.593 \AA .{ }^{18,19} 4,4^{\prime}$-Dimethyl-CHD forms a helical structure similar to that of dimedone. ${ }^{20} 2,5,5^{\prime}$ Trimethyl-CHD forms a hydrate with a four-unit cyclic structure somewhat reminiscent of the $\mathrm{CHD}_{6} \mathrm{Bz}$ complex. ${ }^{18}$ The crystal structure of the 5-methyl derivative is apparently unknown but this compound forms cyclamer complexes with benzene. ${ }^{21}$ These structures have been discussed in review articles and discussions of the nature of hydrogen bonding from the point of view of the "construction" of molecular crystals. ${ }^{22-23}$

We have investigated CHD and its benzene cyclamer complex using IR, Raman, and inelastic incoherent neutron scattering vibrational spectroscopies and have applied density functional theory (DFT) computational methods to various models of these systems. These density functional methods have been applied to oligomeric cluster models of the $\mathrm{CHD}$ linear chain, an infinite one-dimensional linear chain, and the full three-dimensional periodic solid, as well as the benzene cyclamer in $\mathrm{C}_{6}$ symmetry. One of the objectives of this investigation is to assess the reliability of DFT methods with respect to hydrogen-bonded structures and vibrations. Another objective is to understand the structure and dynamics of these interesting materials. The cooperative nature of the hydrogen bond formation and the multiplicity of hydrogen bonding motifs are of particular interest. This cooperative nature of hydrogen bonding is revealed in changes in the computed structure of CHD when it forms hydrogen bonds.

Vibrational spectroscopy coupled to modern ab initio and density functional methods provides detailed information concerning the structure of solids. Inelastic neutron scattering (INS) spectroscopy is a particularly useful method for studies of hydrogen bonding in organic solids because of its sensitivity to $\mathrm{H}$-atom motions. Selective deuteration can be used to eliminate particular components of a material to emphasize the remaining protons. Further, the intensity of a feature in an INS spectrum depends only on the atomic motions in that vibration, not on the response of the electrons to the motions of the nuclei. This makes the calculation of the INS spectrum quite simple, permitting a detailed test of a proposed description of the atomic dynamics by comparison with experiment.

Organic molecular solids such as those formed by hydrocarbons that have very weak intermolecular interactions have molecular structures and vibrations in the solid that are well described by computational treatments of the isolated molecular units. High-resolution vibrational spectroscopy can detect minor effects of these intermolecular interactions that are usually below the resolution of the crystallographic data. In the case of hydrogen-bonded structures, however, both the heavy atom structure and the hydrogen atom dynamics are significantly influenced by intermolecular interactions. In some cases this can be treated in terms of small cluster models that incorporate the first-order effects of hydrogen bonding. Cooperativity of the hydrogen bonding interaction results in the need to consider large clusters. Cooperativity is defined as a property of a system of interacting units (molecules here) in which the energy per unit of a collection of interacting units depends on their state of aggregation. This is usually defined in the sense that the association interaction (energy released by association) increases as the size of the collection increases. In the case of free energy of association, this is usually due to a decrease in the entropy loss of each step as in the formation of an alpha helix from a random coil. In the case of energetic cooperativity, this effect is most likely often due to the influence of prior interactions on the charge distribution of the units as they interact. In the
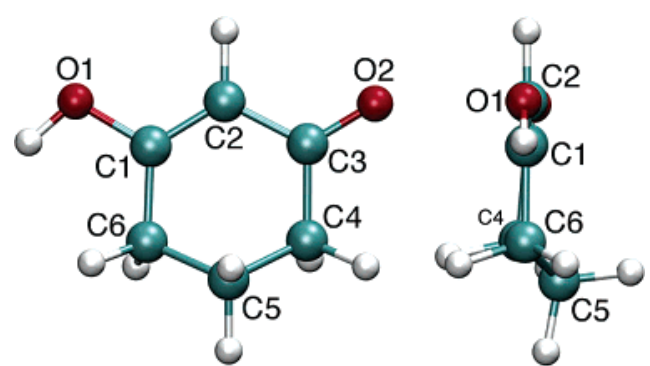

Figure 2. Molecular structure of $\mathrm{CHD}$ at low temperature and the numbering system used (following Katrusiak ${ }^{1}$ ).

present case, this is due to a change in the molecular structure upon hydrogen bonding.

$\mathrm{CHD}$ and its $\mathrm{CHD}_{6} \mathrm{Bz}$ cyclamer differ in their hydrogen bonding motif not only in terms of the local conformation, but also in their nanoscale structure. The linear chain arrangement of the enol of CHD has an infinite scale; the $\mathrm{CHD}_{6} \mathrm{Bz}$ cyclamer is finite in its linkage, permitting a treatment of the entire hydrogen-bonded structure. In effect, cyclic boundary conditions apply. Comparison of these two structures permits separation of the effects of long-range cooperativity on the observed structures and spectra from the local intermolecular interactions involved. This is an alternative to the use of full periodic DFT methods that are also evaluated in the present study.

\section{Experimental and Computational Methods}

INS experiments were carried out at the NIST Center for Neutron Research using the Filter Analyzer Neutron Spectrometer (FANS) on BT4 and the Fermi Chopper Spectrometer $(\mathrm{FCS})^{24}$ on NG6, and also at the ISIS facility of the Rutherford Appleton Laboratory using the time-focusing, crystal analyzer spectrometer TOSCA. ${ }^{25}$ Polycrystalline samples of ca. $1 \mathrm{~g}$ were held at $15 \mathrm{~K}$ for the downscattering experiments, while data taken on FCS were taken with the sample at $200 \mathrm{~K}$ in neutronenergy gain. A more detailed description of the FANS and TOSCA spectrometers and of neutron scattering in general has been presented. ${ }^{26}$

Raman spectra of room-temperature samples were obtained using a Labram Raman spectrometer (Jobin Yvon, Ltd.) equipped with an 1800 grooves/mm holographic grating, a holographic supernotch filter and a Peltier-cooled CCD detector. Samples were excited using a helium-neon laser with an output of $8 \mathrm{~mW}$ at the sample with the $632.8-\mathrm{nm}$ line. The spectrometer was configured with an Olympus BX40 microscope fitted with an Olympus objective having a magnification of $50 \times$ and a numerical aperture of 0.75 . Additional Raman spectra were obtained by macrosampling. Infrared spectra were obtained at room temperature with a Midac FTIR spectrometer with $4 \mathrm{~cm}^{-1}$ spectral resolution.

Geometry optimizations and vibrational analyses were carried out for the CHD clusters and the $\mathrm{CHD}_{6} \mathrm{Bz}$ cyclamer using the Jaguar program ${ }^{27}$ at the B3LYP $28,29 / 6-31 \mathrm{G}^{* * 30}$ level of theory. Periodic DFT calculations of the one-dimensional CHD chain and the CHD unit cell were carried out at the BLYP/dnp level of theory with the program ${ }^{31-33} \mathrm{Dmol}^{3}$ on the SGI Origin Array at the National Center for Supercomputing Applications (NCSA). INS spectra were computed from the normal mode eigenvectors of the harmonic normal-mode analysis resulting from these calculations as previously described and illustrated. ${ }^{26}$

\section{Results}

The molecular structure of CHD obtained from an X-ray study at $213 \mathrm{~K}$ is shown in Figure 2. ${ }^{1}$ The numbering system used is 
TABLE 1: Calculated and Experimental Bond Lengths for Cyclohexanedione and Its Benzene Complex. All Bond Lengths in Angstroms

\begin{tabular}{|c|c|c|c|c|c|c|c|c|c|c|}
\hline \multirow[b]{5}{*}{$\mathrm{N}=$} & \multicolumn{7}{|c|}{ 1,3-cyclohexanedione (CHD) } & \multicolumn{3}{|c|}{ CHD:benzene $6: 1\left(\mathrm{CHD}_{6} \mathrm{Bz}\right)$} \\
\hline & \multicolumn{5}{|c|}{ calculations } & \multicolumn{2}{|c|}{$\mathrm{X}$-ray } & \multirow{4}{*}{$\begin{array}{c}\text { calc. } \\
\text { Jaguar } \\
\mathrm{C}_{6}\end{array}$} & \multirow{3}{*}{$\begin{array}{c}\text { X-ray } \\
\text { Etter et al. }\end{array}$} & \\
\hline & \multirow{2}{*}{\multicolumn{3}{|c|}{$\begin{array}{c}\text { linear cluster of size } \mathrm{N} \\
\text { Jaguar B3LYP/6-31G** }\end{array}$}} & \multicolumn{2}{|c|}{ infinite system } & \multirow{3}{*}{$\frac{\text { Etter et al. }^{14}}{298 \mathrm{~K}}$} & \multirow{3}{*}{$\frac{\text { Katrusiak }^{1}}{213 \mathrm{~K}}$} & & & \\
\hline & & & & $\mathrm{DMol}^{1}$ & $\mathrm{DMol}^{3}$ & & & & & \\
\hline & 3 & 5 & 7 & $\overline{S=13.78}$ & & & & & $298 \mathrm{~K}$ & \\
\hline $\mathrm{C} 1-\mathrm{O} 1$ & 1.336 & 1.330 & 1.325 & 1.331 & 1.332 & 1.323 & 1.327 & 1.316 & 1.318 & $\mathrm{C} 1-\mathrm{O} 1$ \\
\hline $\mathrm{C} 3-\mathrm{O} 2$ & 1.241 & 1.240 & 1.247 & 1.267 & 1.270 & 1.243 & 1.245 & 1.254 & 1.253 & $\mathrm{C} 3-\mathrm{O} 2$ \\
\hline $\mathrm{C} 1-\mathrm{C} 2$ & 1.361 & 1.365 & 1.368 & 1.381 & 1.380 & 1.345 & 1.351 & 1.377 & 1.349 & $\mathrm{C} 1-\mathrm{C} 2$ \\
\hline $\mathrm{C} 2-\mathrm{C} 3$ & 1.448 & 1.441 & 1.437 & 1.430 & 1.426 & 1.409 & 1.417 & 1.432 & 1.413 & $\mathrm{C} 2-\mathrm{C} 3$ \\
\hline $\mathrm{C} 3-\mathrm{C} 4$ & 1.531 & 1.526 & 1.523 & 1.529 & 1.519 & 1.49 & 1.508 & 1.524 & 1.512 & $\mathrm{C} 3-\mathrm{C} 4$ \\
\hline $\mathrm{C} 4-\mathrm{C} 5$ & 1.532 & 1.531 & 1.531 & 1.540 & 1.532 & & 1.514 & 1.531 & 1.490 & $\mathrm{C} 4-\mathrm{C} 5$ \\
\hline $\mathrm{C} 5-\mathrm{C} 6$ & 1.534 & 1.533 & 1.532 & 1.541 & 1.541 & & 1.522 & 1.530 & 1.495 & $\mathrm{C} 5-\mathrm{C} 6$ \\
\hline \multirow[t]{2}{*}{$\mathrm{C} 6-\mathrm{C} 1$} & 1.510 & 1.511 & 1.510 & 1.514 & 1.509 & 1.483 & 1.496 & 1.507 & 1.513 & $\mathrm{C} 6-\mathrm{C} 1$ \\
\hline & \multicolumn{4}{|c|}{ RMSD calculated vs X-ray } & & \multicolumn{2}{|c|}{$\begin{array}{l}\text { RMSD of X-ray } \\
\text { structures }\end{array}$} & \multicolumn{2}{|c|}{$\begin{array}{c}\text { RMSD calculated vs } \\
\text { X-ray }\end{array}$} & \\
\hline $298 \mathrm{~K}$ & 0.027 & 0.024 & 0.023 & 0.028 & 0.025 & 0.010 & & 0.023 & & \\
\hline \multirow[t]{2}{*}{$213 \mathrm{~K}$} & 0.017 & 0.015 & 0.013 & 0.021 & 0.018 & & & & & \\
\hline & & hydr & en bond s & metry $(\mathrm{OH}$ & : anti-ant & & & & syn-anti & \\
\hline $\mathrm{O} 1-\mathrm{H}$ & 0.944 & 1.001 & 1.007 & 1.036 & 1.044 & 0.94 & 0.996 & 1.027 & 1.010 & $\mathrm{O} 1-\mathrm{H}$ \\
\hline $\mathrm{O} 2^{\prime} \angle \mathrm{H}$ & 1.671 & 1.620 & 1.582 & 1.550 & 1.518 & 1.63 & 1.580 & 1.513 & 1.570 & $\mathrm{O} 2-\mathrm{H}$ \\
\hline $\mathrm{O} 1-\mathrm{O} 2^{\prime}$ & 2.665 & 2.621 & 2.589 & 2.585 & 2.561 & 2.561 & 2.576 & 2.540 & 2.579 & $\mathrm{O} 1-\mathrm{O} 2$ \\
\hline $\mathrm{O}-\mathrm{H}-\mathrm{O}$ & (178.6) & (178.6) & (178.6) & 177.63 & 177.41 & 170 & 179.3 & 177.1 & 174 & $\mathrm{O}-\mathrm{H}-\mathrm{O}$ \\
\hline
\end{tabular}

the one chosen uniformly below. (This is not the standard numbering for the enol. Its choice is because the positions of the protons involved in the intermolecular hydrogen bond switch when the sample is cooled through a phase transition.) A comparison of the results of the various DFT structural calculations with the results of X-ray diffraction studies for both the CHD crystal and its cyclamer complex with benzene are summarized in Table 1 . The heavy atom bond lengths are shown first, followed by values for the root-mean-square standard deviations (RMSD) of these calculated values relative to the two crystal structures. The hydrogen bonding geometry is presented at the bottom of Table 1 .

In the calculation for the $\mathrm{CHD}_{6} \mathrm{Bz}$ complex, the geometry was assumed to conform to $\mathrm{C}_{6}$ symmetry. The actual crystal symmetry is $\mathrm{S}_{6}$ with the alternate $\mathrm{C} 5$ atoms above and below the plane. It was felt that this difference in symmetry would have at most a very minor influence on the computed spectra. This isolated cyclamer unit was treated as a supermolecule starting from the geometry observed in the crystal. The resulting RMSD for the bond lengths involving the heavy atoms is 0.023 $\AA$ A.

Calculations at the B3LYP/6-31G** level of theory were performed for linear cluster models of the CHD linear chain composed of 3,5 , and 7 units. In these clusters the individual units were constrained to have identical internal geometry. All $\mathrm{O} 1-\mathrm{O} 2^{\prime}$ distances were constrained to be the same for each of the $\mathrm{N}-1$ hydrogen bonds in the cluster and the $\mathrm{O} 1-\mathrm{H}-\mathrm{O} 2^{\prime}$ bond angle was constrained to be that observed in the crystal, $178.6^{\circ}$. Two crystal structures of CHD will be discussed. The first is that obtained at $298 \mathrm{~K}$ by Etter et al. ${ }^{14}$ and the second at $213 \mathrm{~K}$ by Katrusiak. ${ }^{1}$ This latter author has investigated an interesting solid-solid, order-disorder phase transition of CHD as a function of temperature and pressure. The transition involves the out-of-plane position of the $\mathrm{C} 5$ atom with respect to the planar, hydrogen bonded system of $\mathrm{C} 1, \mathrm{C} 2, \mathrm{C} 3, \mathrm{C} 4, \mathrm{C} 6, \mathrm{O} 1$, and $\mathrm{O} 2$. The transition temperature is $289 \mathrm{~K}$. At the higher temperature the $\mathrm{C} 5$ carbon is disordered, with occupancy above and below the plane of the other five atoms of the ring. At low temperature the structure becomes that shown in Figure 2. A cooperative change in the position of the hydrogen bonding proton also occurs at this phase transition. Because of the $\mathrm{C} 5$ disorder at room temperature, the bond distances to the neighboring atoms are somewhat in question and these are omitted from Table 1. With this exclusion, the RMSD for the two X-ray structures from each other is $0.010 \AA$. The RMSD for the structures calculated for the cluster models from each diffraction result is given in Table 1 for the heavy atoms only.

The results for the periodic DFT calculations are also given in Table 1 in the same format. The entry $\mathrm{DMol}^{1}$ refers to an infinite, one-dimensional linear chain with two molecules forming the repeat unit. The period for the $213 \mathrm{~K}$ unit cell parameters is $13.855 \AA .{ }^{1}$ Optimized structures were computed for one-dimensional chain periodicities ranging from 13.9 to 13.6 ̊. The DMol program does not optimize lattice constants as part of the general geometry optimization procedure, necessitating the stepwise adjustment of the repeat distance to determine the minimum energy spacing. The energy as a function of the periodicity was found to have a minimum at $13.78 \AA$. This diminution of lattice parameter may be a consequence of removing the intermolecular forces perpendicular to the infinite linear chains that occur in the complete unit cell, the slight contraction of the linear chain from the $213 \mathrm{~K}$ unit cell spacing to what is otherwise a $0 \mathrm{~K}$ energy minimized structure, small differences between the calculated and crystal geometries that add to a net decrease in lattice spacing, or some combination of all three. At this periodicity the energy is lower than at $13.855 \AA$ by approximately $15 \mathrm{~cm}^{-1}$. The structure calculated for the repeat of $13.78 \AA$ is given in Table 1 . The $\mathrm{DMol}^{3}$ entry in Table 1 corresponds to a simulation of the full three-dimensional unit cell structure using the reported crystal lattice parameters determined at $213 \mathrm{~K}^{1}$, where the above considerations may also be taken in comparing the calculated $(0 \mathrm{~K})$ unit cell properties with the $213 \mathrm{~K}$ crystal structure.

The INS spectra for the $\mathrm{CHD}_{6} \mathrm{Bz}$ complex and for crystalline CHD are shown in Figures 3 and 4. In Figure 3 the INS spectrum of the $\mathrm{CHD}_{6} \mathrm{Bz}$ complex is compared with Raman and IR spectra. In Figure 4 the INS spectrum of the pure CHD is compared with that for $\mathrm{CHD}_{6} \mathrm{Bz}-d_{6}$. Figure 5 is a comparison of the INS spectrum of the $\mathrm{CHD}_{6} \mathrm{Bz}$ complex with the results of a simulation of the INS spectrum based on a harmonic analysis of the energy-minimized structure compared to the $\mathrm{X}$-ray results in Table 1. Figure 6 shows the low-frequency 


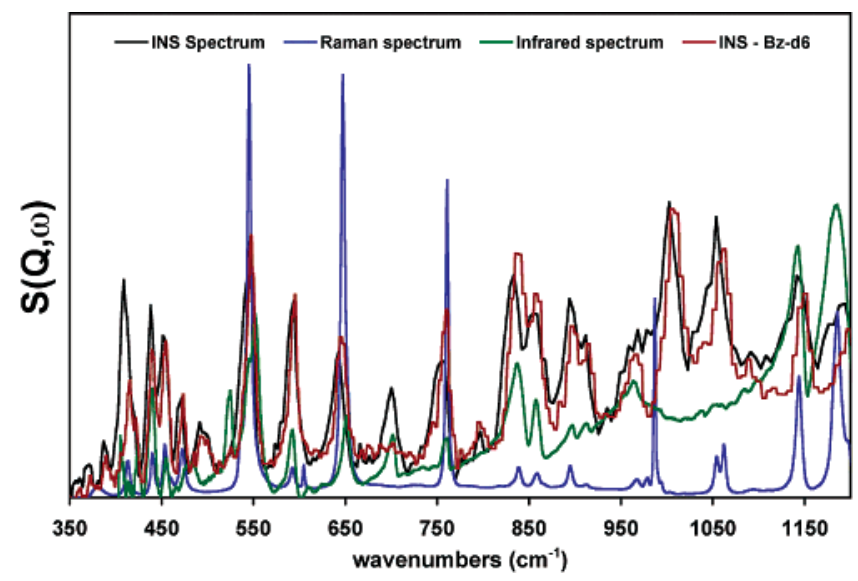

Figure 3. The INS, IR, and Raman spectra of $\mathrm{CHD}_{6} \mathrm{Bz}$ and the INS spectrum of $\mathrm{CHD}_{6} \mathrm{Bz}-d_{6}$.

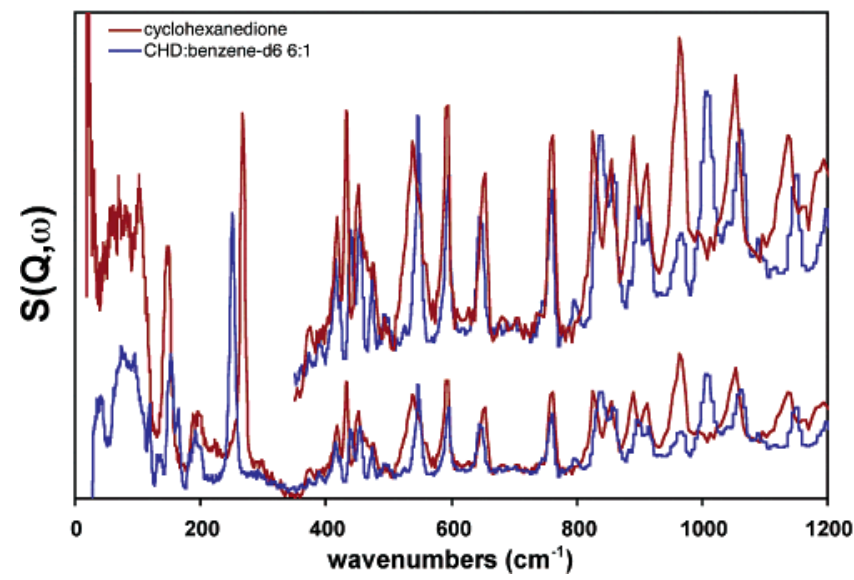

Figure 4. Comparison of the INS spectra of $\mathrm{CHD}$ and $\mathrm{CHD}_{6} \mathrm{Bz}-d_{6}$.

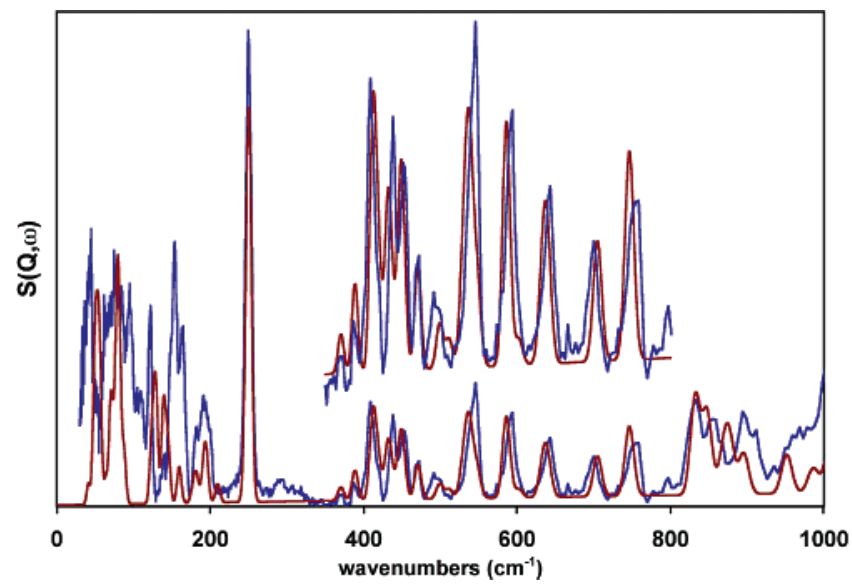

Figure 5. Comparison of the calculated and observed INS spectra of $\mathrm{CHD}_{6} \mathrm{Bz}$. The calculation is a $\mathrm{B} 3 \mathrm{LYP} / 6-31 \mathrm{G}^{* *}$ calculation performed by Jaguar. ${ }^{27}$ Calculation in red; experiment in blue.

region of this spectrum for $\mathrm{CHD}_{6} \mathrm{Bz}$ obtained with TOSCA at ISIS, the Fermi Chopper spectrometer at NCNR, and the spectrum computed from the cyclamer model. Figure 7 is a comparison of the INS spectrum of CHD with the calculated INS spectra based on a harmonic treatment of an infinite periodic one-dimensional chain structure $\left(\mathrm{DMol}^{1}\right)$, the optimized pentamer, and the three-dimensional periodic treatment of the unit cell $\left(\mathrm{DMol}^{3}\right)$ of Table 1 .

\section{Discussion}

The spectra of Figure 3 clearly identify the $\mathrm{C}_{6} \mathrm{H}_{6}$ features in the complex with CHD. This includes the inactive (by optical

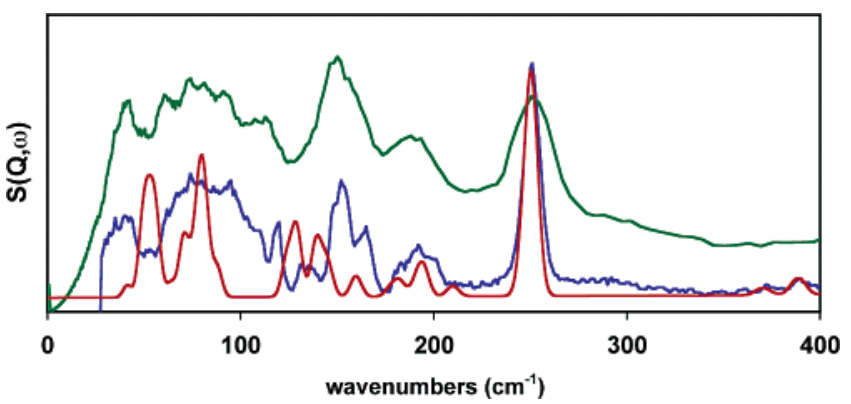

Figure 6. Expanded version of the INS spectrum of $\mathrm{CHD}_{6} \mathrm{Bz}$ in the low-frequency region with addition of FCS data for a sample temperature of $200 \mathrm{~K}$. FCS, green; TOSCA, blue; calc, red.

methods) $\mathrm{e}_{2 \mathrm{u}}$ mode $v_{10}{ }^{34}$ at $400 \mathrm{~cm}^{-1}$, the IR active $\mathrm{a}_{2 \mathrm{u}}$ mode $v_{11}$ near $700 \mathrm{~cm}^{-1}$, and the Raman active modes $v_{10}\left(\mathrm{e}_{1 \mathrm{~g}}\right)$ and $v_{1}\left(\mathrm{a}_{1 \mathrm{~g}}\right)$ near 840 and $990 \mathrm{~cm}^{-1}$, respectively. In most cases these features in the $\mathrm{CHD}_{6} \mathrm{Bz}$ complex disappear in the INS spectrum of the $\mathrm{CHD}_{6} \mathrm{Bz}-d_{6}$ complex due to the relative inactivity of the deuterium nucleus as a scattering center. On this basis alone, one can also identify the benzene $v_{18} \mathrm{e}_{1 \mathrm{u}}$ mode at $1037 \mathrm{~cm}^{-1}$, the $1146 \mathrm{~cm}^{-1} \mathrm{~b}_{2 \mathrm{u}}$ mode $v_{15}$, and the $1178 \mathrm{~cm}^{-1}$ $\mathrm{e}_{2 \mathrm{~g}}$ mode $v_{9}$. In some cases, for example, the $v_{10} \mathrm{e}_{1 \mathrm{~g}}$ mode, there is no appreciable decrease in the INS intensity upon deuteration due, presumably, to overlap by dione vibrations. In such cases, the assignment is based on the IR and Raman spectra and the known assignments for benzene.

Comparison of the spectrum of $\mathrm{CHD}$ with that of the $\mathrm{CHD}_{6}$ $\mathrm{Bz}-d_{6}$ cyclamer complex in Figure 4 reveals the effects of the different hydrogen bonding motifs and the intermolecular packing in these two structures. The major differences are seen in the region near $250 \mathrm{~cm}^{-1}$, near $435 \mathrm{~cm}^{-1}$, and in the 950 to $1050 \mathrm{~cm}^{-1}$ region. The strong band near $250 \mathrm{~cm}^{-1}$ is due to wagging of the $\mathrm{CH}_{2}$ group at $\mathrm{C} 5$. The $435 \mathrm{~cm}^{-1}$ region is due to out-of-plane motions of the $\mathrm{C} 4$ and $\mathrm{C} 6$ atoms. The feature near $1025 \mathrm{~cm}^{-1}$ in the $\mathrm{CHD}_{6} \mathrm{Bz}-d_{6}$ complex is due to out-ofplane motions of the $\mathrm{OH}$ groups. The nearby features of the pure $\mathrm{CHD}$ crystal are due to $\mathrm{CH}$ wagging motions of the $\mathrm{C} 5$ atoms admixed with multiple other degrees of freedom.

The comparison of the observed INS spectrum of the $\mathrm{CHD}_{6} \mathrm{Bz}$ cyclamer complex with that computed on the basis of a full $\mathrm{C}_{6}$ symmetry B3LYP/6-31G** calculation is shown in Figure 5. The agreement is taken to be excellent especially considering that only fundamental transitions are included in this calculation. The only region of significant disagreement is in the very low frequency region where phonon activity occurs. Phonons are not included in the simulation used here but some of the lowfrequency modes are, of course, due to flexing of the cyclamer and are included. This is shown in an expanded view in Figure 6. This figure includes data obtained at $200 \mathrm{~K}$ from the Fermi Chopper Spectrometer at NIST.

Figure 7 shows the DFT calculations for the pentamer model, the two periodic calculations, and the case of CHD itself. The pentamer and periodic $\left(\mathrm{DMol}^{1}\right.$ and $\left.\mathrm{DMol}^{3}\right)$ models are roughly equally good in reproducing the experimental observations, with the pentamer and $\mathrm{DMol}^{3}$ unit cell models being a slightly better representation of the data overall than the isolated hydrogenbonded chain model $\left(\mathrm{DMol}^{1}\right)$. It is clear that neither of these representations of the data is as good as the case for the benzene cyclamer complex. Most of the features are properly reproduced but at slightly shifted frequencies. This is most apparent for the very large feature near $250 \mathrm{~cm}^{-1}$. Examination of Figure 3 shows that this vibration, which is primarily a wagging motion of the $\mathrm{CH}_{2}$ group at $\mathrm{C} 5$, is shifted to higher frequency in the 


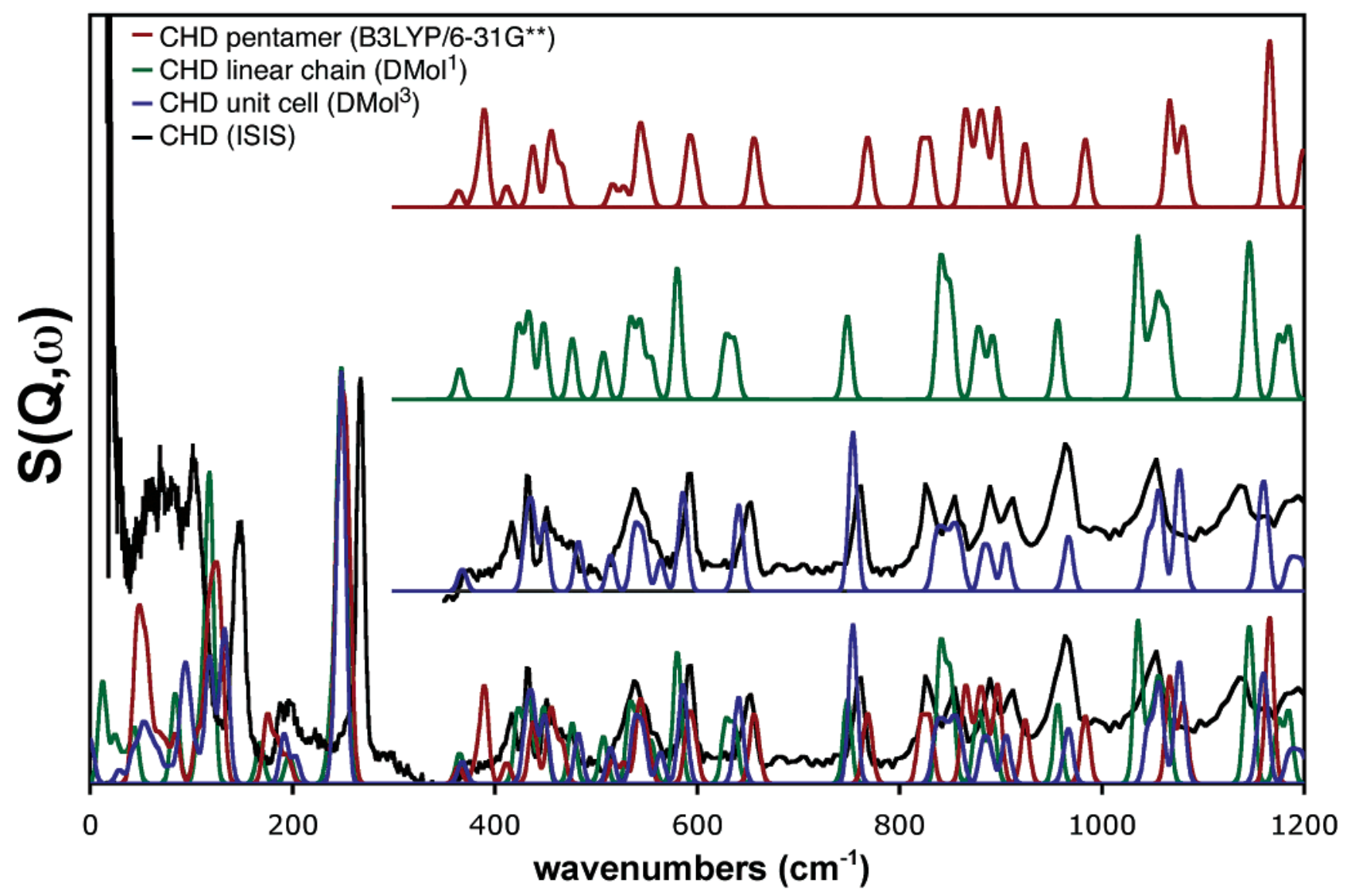

Figure 7. Comparison of the observed and calculated INS spectra of CHD.

neat material compared to that of the benzene complex, but that this shift is not revealed in any of the calculations. The ordering of this $\mathrm{CH}_{2}$ group is one of the features associated with the phase transition that occurs at $289 \mathrm{~K}$. This phase transition is due to increasingly dense packing at lower temperature. This suggests that the aspect of the real crystal that is not properly treated in these computations is this crystal-packing force acting on $\mathrm{C} 5$. In the finite chain and one-dimensional $\mathrm{DMol}^{1}$ calculations this interaction is missing. In the $\mathrm{DMol}^{3}$ unit cell calculation this interaction is included, but the repulsive intermolecular interaction appears to not affect the frequency of the motion. This may be either a limit of the DFT method or a result of the $213 \mathrm{~K}$ unit cell parameters being employed for a $0 \mathrm{~K}$ vibrational analysis in the absence of any lattice parameter adjustment. While the $213 \mathrm{~K}$ unit cell is below the orderdisorder phase transition and the C5 positions are fixed, it is conceivable that the continued volume reduction of the unit cell at lower temperatures (such as the $15 \mathrm{~K}$ INS study) increases the repulsive interaction enough to shift the frequency of this mode.

We turn now to an examination of the systematic variation of the calculated structure with the number of units included. The main structural features of interest are the $\mathrm{O}-\mathrm{O}^{\prime}$ intermolecular hydrogen bonding distance and the difference in the $\mathrm{C}_{23}$ and $\mathrm{C}_{12}$ bond lengths. This latter quantity is the difference in bond length between the nominal single bond and the nominal double bond in the conjugated system. Gilli and co-workers ${ }^{7-11}$ have pointed out that it is expected that these two quantities are correlated. Examination of the crystal structures of various similar molecules indicates that this is the case. This is a consequence of what has been designated "Resonance Assisted Hydrogen Bonding." The origin of this behavior becomes clear if we consider an $\mathrm{O}-\mathrm{O}^{\prime}$ distance that is sufficiently small that the intervening $\mathrm{H}$ nucleus is centered between these atoms. In that case, there is no structural difference between the $C_{23}$ and $\mathrm{C}_{12}$ bonds due to symmetry and so the difference $\mathrm{R}_{23}-\mathrm{R}_{12}$ vanishes.

Figure $8 \mathrm{~A} \& \mathrm{~B}$ display the variation of the $\mathrm{O}-\mathrm{O}^{\prime}$ and $\mathrm{R}_{23}-$ $\mathrm{R}_{12}$ difference as a function of $1 / N$ where $N$ is the number of units in the model treated. For this construction, the crystal and the benzene cyclamer are treated as having an infinite number of units. In Figure $8 \mathrm{C}$ the correlation between these two quantities is displayed. The line is drawn so as to fit the finite cluster computed results (open squares). Both the $\mathrm{O}-\mathrm{O}^{\prime}$ distance and $R_{23}-R_{12}$ difference extrapolate close to the value computed for the cyclamer. The linear extrapolation shown in Figure 8 for the variation in the $\mathrm{O}-\mathrm{O}^{\prime}$ distance and quadratic extrapolation for the $\mathrm{R}_{23}-\mathrm{R}_{12}$ distance results in coincidence with the value computed for the cyclamer. This indicates that the theoretical model is internally consistent and that the variation in these quantities is a result of the change in the number of interacting units used in the model.

The $\mathrm{O}-\mathrm{O}^{\prime}$ distance extrapolated from the finite models or computed for the cyclamer is smaller than the values deduced from the X-ray diffraction data by 0.02 (Etter) to 0.03 (Katrusiak) $\AA$. The $\mathrm{DMol}^{1}$ one-dimensional infinite linear chain computation is closer to the experimental observations $(\sim 0.01$ (Etter) to $\sim 0.02$ (Katrusiak) $\AA$ ). The value of $2.561 \AA$ obtained from the $\mathrm{DMol}^{3}$ computation is in exact agreement with the experimental value for the room-temperature structure of 2.561 $\AA$. Two significant issues arise in making this comparison, however. The first is that the value of the $\mathrm{O}-\mathrm{O}^{\prime}$ distance determined from the X-ray data depends on the sample temperature. The $\mathrm{O}-\mathrm{O}^{\prime}$ distance is $2.576 \AA$ at $213 \mathrm{~K}$, somewhat shorter than at room temperature. This variation is the origin of the range of values in the comparisons made above. The 
(A)

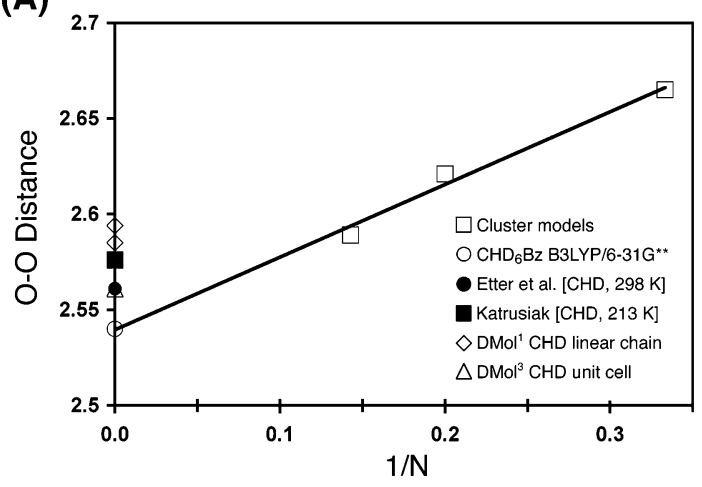

(B)

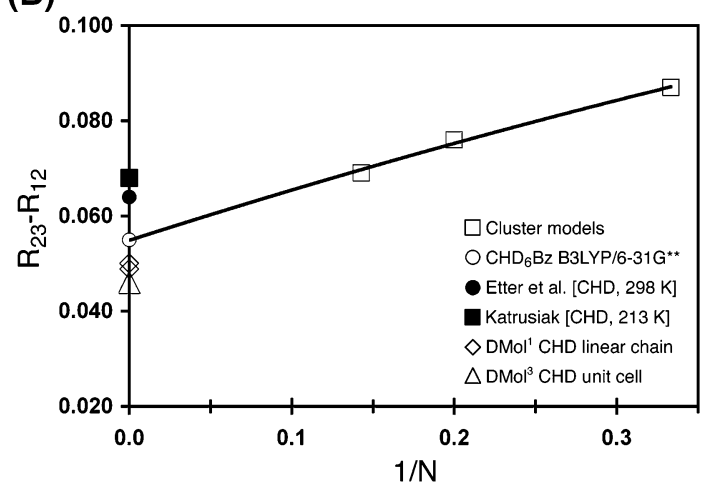

(C)

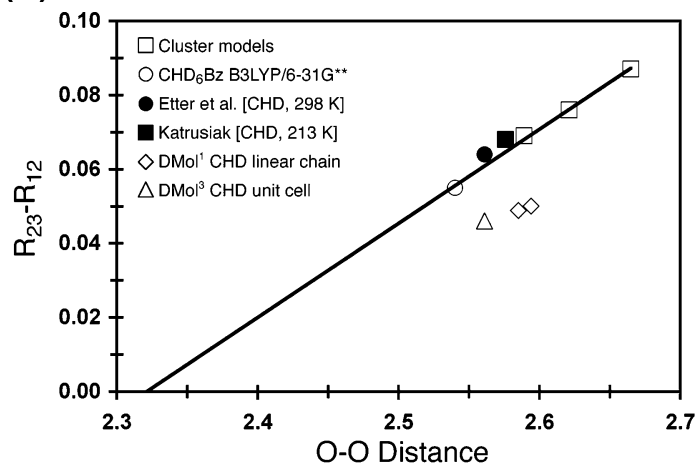

Figure 8. Trends in structural changes. (A) Variation of the hydrogen bonding $\mathrm{O}-\mathrm{O}$ distance with the reciprocal of the chain length and comparison with infinite chain calculations and experiment. The two values for the one-dimensional $\mathrm{DMol}^{1}$ calculations correspond to different values of the periodic limits with the upper point being for $\mathrm{S}$ $=13.855 \AA$ unit cell spacing and the lower point being for the optimized unit cell spacing where $S=13.78 \AA$. (B) Variation in the difference in carbon-carbon bond lengths with reciprocal cluster length compared to experiment and infinite chain calculations. (C) Variation in carboncarbon bond length difference with $\mathrm{O}-\mathrm{O}$ hydrogen bonded distance. The left and right points for the $\mathrm{DMol}^{1}$ calculation are for the two periodic spacing values of (A).

second issue is that the value obtained from a periodic calculation depends on the periodicity used for that calculation. In the present case the $\mathrm{DMol}^{3}$ calculation was performed using the lattice parameters obtained at $213 \mathrm{~K}$, making the target distance the experimental value of $2.576 \AA$. Since the periodicity used is that of the $213 \mathrm{~K}$ crystal, the $0.015 \AA$ shortening of the $\mathrm{O}-\mathrm{O}^{\prime}$ value is due to the fact that optimization of the molecular structure in the lattice results in a molecule that is overall slightly larger than is experimentally observed along the line of the hydrogen bonding chain and cannot be attributed to failure of the method to reproduce the hydrogen bond per se.
The series of computations performed for the one-dimensional periodic chain can be used to examine the effect of the periodicity on the $\mathrm{O}-\mathrm{O}^{\prime}$ distance. These calculations show that a change in the one-dimensional lattice spacing results in a change in the $\mathrm{O}-\mathrm{O}^{\prime}$ distance that is linear in the lattice variation with a slope of 0.23 . A change of the lattice period of $0.1 \AA$ results in a change in the $\mathrm{O}-\mathrm{O}^{\prime}$ distance of only $0.023 \AA$. This means that when the spacing between molecules increases, the molecule expands to take up $77 \%$ of the extension of the $\mathrm{O}-\mathrm{O}^{\prime}$ separation.

Another complication is that the three-dimensional $\mathrm{DMol}^{3}$ unit cell calculation was conducted with a lattice spacing determined from data at $213 \mathrm{~K}$ while the calculation corresponds to $0 \mathrm{~K}$. The trend, based on two data points, is toward a longer $\mathrm{O}-\mathrm{O}^{\prime}$ distance at lower temperature. The magnitude of this empirical trend is more than enough to account for any difference between the computed and observed $\mathrm{O}-\mathrm{O}^{\prime}$ distances.

The bond length difference $\mathrm{R}_{23}-\mathrm{R}_{12}$ also varies with chain length in the calculations (see Figure $8 \mathrm{~B}$ ). The finite chain values extrapolate to the value computed for the benzene cyclamer. This cyclamer value is, in turn, in good agreement with the value computed using $\mathrm{DMol}^{3}$ for the periodic solid. The $\mathrm{R}_{23}-$ $\mathrm{R}_{12}$ values for the $\mathrm{DMol}^{1}(0.049 \AA), \mathrm{DMol}^{3}(0.046 \AA)$, and cyclamer $(0.055 \AA)$ calculations are somewhat smaller than the values observed, which are $0.064-0.066 \AA$. This may reflect the well-known overemphasis of DFT on delocalization.

A plot of $\mathrm{R}_{23}-\mathrm{R}_{12}$ vs. $\mathrm{O}-\mathrm{O}^{\prime}$ distance is shown in Figure $8 \mathrm{C}$. It is interesting to see at what value of the $\mathrm{O}-\mathrm{O}^{\prime}$ distance this correlation extrapolates to a zero value for the bond length difference. This should correspond to the $\mathrm{O}-\mathrm{O}^{\prime}$ distance at which the proton is equally shared and thus the factor distinguishing one $\mathrm{C}-\mathrm{O}$ bond from the other disappears. A linear extrapolation gives a value near $2.3 \AA$ if only the computed finite cluster values are used. This is shorter than the value of ca. $2.4 \AA$ expected on the basis of other $\mathrm{O}-\mathrm{O}$ hydrogen bonding distances involving short strong, symmetric hydrogen bonds. ${ }^{35-39}$ This extrapolation may well be non-linear, however, so the intercept is at a longer $\mathrm{O}-\mathrm{O}$ distance. In any case the trend of the effect of increasing the length of the hydrogen bonded chain on the internal structure of the CHD unit appears to agree qualitatively with expectations based on short-strong symmetric hydrogen-bonded systems. The cooperative effect of the hydrogen bonding on the internal structure of the molecule has a trend whose magnitude is consistent with the placement of atoms so that the hydrogen bonding is stronger.

\section{Conclusions}

The very good to excellent agreement between the observed and computed inelastic neutron scattering spectra and molecular structures for 1,3-cyclohexanedione and its complex with benzene implies that the computational methods used provide a reasonably accurate quantitative description of this hydrogenbonded system. Given the uncertainties introduced by finite temperature and experimental uncertainty, there seems to be good reason to believe that these computations are reliable. This extends to the simulations of the finite clusters that are not amenable to experimental observations. Examination of the energies of the trimer, pentamer, and heptamer cluster models shows that the pentamer is more stable than expected on the basis of the trimer and that the heptamer is even more stable. The cooperative stabilization energy per unit is on the order of a few $\mathrm{kcal} / \mathrm{mol}$ and decreases on going from pentamer to heptamer. This is the trend expected since for long chains the incremental energy should approach a constant. The dipole 
moment per unit increases from 7.7 to 8.8 to $9.5 \mathrm{D}$ in going from trimer to pentamer to heptamer, revealing the increased contribution of the ionic resonance form induced by hydrogen bond formation. Both of these trends support the conclusion that the association of the CHD units is a "cooperative phenomenon."

On this basis we come to the following conclusions.

(a) The atom-centered periodic generalized gradient approximation (GGA) DFT method used $\left(\mathrm{DMol}^{3}\right)$ provides an adequate description of this hydrogen-bonded solid. This conclusion does not yet encompass the potential energy for the motion of the proton involved in the hydrogen bond between the two $\mathrm{O}$ atoms to which it is bonded, since this degree of freedom has not yet been identified in the experimental spectrum. This is primarily due to the fact that this motion is most probably in a higher frequency region where it is reduced in intensity in this spectrometer and overlapped by overtone and combination transitions. Direct geometry chopper spectrometers with lower momentum transfer can perhaps be used to identify this motion.

(b) The comparison of computed and observed structures and vibrational spectra for hydrogen-bonded solids using periodic DFT methods requires consideration of the effect of the periodicity of the solid on the computation. Comparison of structural features for calculated and observed structures may be complicated by compensation of the molecule to remove stress induced by the strain of the period not corresponding to that for which the computation would give a minimum energy.

(c) Hydrogen bonding of the enol form of CHD results in a cooperative change in the structure of this molecule in a direction that is expected to result in strengthening of hydrogen bonds in a sequential fashion. This effect has been discussed for the specific case of CHD in previous theoretical work. ${ }^{12,13}$ Here we show that cluster calculations converge to the infinite chain theoretical results and are in agreement with several experimental results. This structural deformation is in the direction expected for resonance enhanced hydrogen bonding. It continues as the cluster extends to and beyond seven molecular units in a linear chain.

(d) This conclusion has the implication that computational methods aimed at predicting crystal packing that are based on isolated molecules are not likely to succeed in such cases.

(e) Behavior of this type is expected for the analogous case of peptides as has been discussed previously on the basis of theoretical calculations ${ }^{40-47}$ and experiments. ${ }^{48-50}$ This is an important consideration in understanding the stability of globular proteins.

Acknowledgment. The Rutherford Appleton Laboratory is thanked for neutron beam access at the ISIS Facility where the TOSCA spectrometer was used. The NIST Center for Neutron Research is thanked for access to the FANS and FCS spectrometers at the NCNR. This work was supported by US National Science Foundation grant CHE 0240104 and by the US Department of Energy grant DE-FG02-01ER14245. The National Center for Supercomputing Applications, University of Illinois at Urbana-Champaign, is thanked for access to the SGI Origin Array for the $\mathrm{DMol}^{3}$ calculations.

\section{References and Notes}

(1) Katrusiak, A. Structure and Phase Transition of 1,3-Cyclohexanedione Crystals as a Function of Temperature. Acta Crystallogr. 1991, B47, 398-404.

(2) Bobrowicz, L.; Katrusiak, A.; Nawrocik, W.; Wasicki, J.; Natkaniec, I. Neutron Scattering Study of the Phase Transition in 1,3-Cyclohexanedione Crystals at Ambient and High Pressures. Phys. Status Solidi B 1996, 196, 39-47.
(3) Katrusiak, A. High-Pressure X-ray Diffraction Study on the Structure and Phase Transition of 1,3-Cyclohexanedione Crystals. Acto Crystallogr. 1990, B46, 246-256.

(4) Pajak, Z.; Latanowicz, L.; Katrusiak, A. NMR Study of Molecular Motions in 1,3-Cyclohexanedione. Phys. Status Solidi A 1992, 130, 421438.

(5) Szafranski, M.; Czarnecki, P.; Katrusiak, A.; Habrylo, S. DTA investigations of phase transitions in 1,3-cyclohexanedione under high pressures. Solid State Commun. 1991, 82, 277-281.

(6) Katrusiak, A. Stereochemistry and transformation of $-\mathrm{OH} \cdots \mathrm{O}=$ hydrogen bonds Part I. Polymorphism and phase transitions of 1,3cyclohexanedione crystals. J. Mol. Struct. 1992, 269, 329-354.

(7) Bertolasi, V.; Gilli, P.; Valeria, F.; Gilli, G. Intramolecular O-H• . $\mathrm{O}$ hydrogen bonds assisted by resonance. Correlation between crystallographic data and ${ }^{1} \mathrm{H}$ NMR chemical shifts. J. Chem. Soc., Perkin Trans. 2, 1997, 945-952.

(8) Gilli, P.; Ferretti, V.; Bertolasi, V.; Gilli, G. A novel approach to hydrogen bonding theory. Adv. Mol. Struct. Res. 1996, 2, 67-102.

(9) Bertolasi, V.; Gilli, P.; Ferretti, V.; Gilli, G. Resonance-assisted $\mathrm{O}-\mathrm{H} \cdots \mathrm{O}$ hydrogen bonding: its role in the crystalline self-recognition of $\beta$-diketone enols and its structural and IR characterization. Chem.-Eur. J. 1996, 2, 925-934.

(10) Gilli, P.; Bertolasi, V.; Ferretti, V.; Gilli, G., Covalent Nature of the Strong Homonuclear Hydrogen Bond. Study of the $\mathrm{O}-\mathrm{H}-\mathrm{O}$ System by Crystal Structure Correlation Methods. J. Am. Chem. Soc. 1994, 116, 909-915.

(11) Gilli, G.; Bertolasi, V.; Ferretti, V.; Gilli, P. Resonance-Assisted Hydrogen Bonding. III. Formation of Intermolecular Hydrogen-Bonded Chains in Crystals of Diketone Enols and its Relevance to Molecular Association. Acta Crystallogr. 1993, B49, 564-576.

(12) Turi, L.; Dannenberg, J. J. Molecular Orbital Study of Crystalline 1,3-Cyclohexanedione. 2. Aggregates in Two and Three Dimensions. Chem. Mater. 1994, 6, 1313-1316.

(13) Turi, L.; Dannenberg, J. J. Molecular Orbital Studies of Crystal Formation: The Aggregation and Nucleation of 1,3-Diones. J. Phys. Chem. 1992, 96, 5819-5824.

(14) Etter, M. C.; Urbanczyk-Lipkowska, Z.; Jahn, D. A.; Frye, J. S, Solid-state structural characterization of 1,3-cyclohexanedione and of a 6 : 1 cyclohexanedione-benzene cyclamer complex, a novel host-guest species. J. Am. Chem. Soc. 1986, 108, 5871-6.

(15) Katrusiak, A Structure of 1,3-cyclopentanedione. Acta Crystallogr., Sect. C 1990, C46, 1289-93.

(16) Katrusiak, A. Structure of 2-Methyl-1,3-cyclopentanedione. Acta Crystallogr., Sect. C 1989, C45, 1897-99.

(17) Katrusiak, A. Conformational Transformation Coupled with the Order-Disorder Phase-Transition in 2-Methyl-1,3-Cyclohexanedione Crystals. Acta Crystallogr. 2000, B 56, 872-881 (See also ibid, 2000, B 56 , 1112).

(18) Semmingsen, D. The Crystal and Molecular Structure of Dimedone Acta Chem. Scand. 1974, B28, 169-174.

(19) Singh, I.; Calvo, C. The Crystal Structure of Dimedone. Can. J. Chem. 1975, 53, 1046-1050.

(20) Barnes, J. C. Hydrogen Bonding in 4,4-Dimethylcyclohexane-1,3dione and 1,5,5-Trimethylcyclohexane-1,3-dione Monohydrate. Acta Crystallogr. 1996, C52, 3159-3162.

(21) Etter, M. C.; Parker, D. L.; Ruberu, S. R.; Panunto, T. W.; Britton, D. Solid-State and Inclusion Properties of Hydrogen-Bonded 1,3-Cyclohexanedione Cyclamers. J. Inclusion Phenom. Mol. Recognit. Chem. 1990, $8,395-407$.

(22) Chin, D. N.; Zerkowski, J. A.; MacDonald, J. C.; Whitesides, G. M. Strategies for the Design and Assembly of Hydrogen-bonded Aggregates in the Solid State. In Organised Molecular Assemblies in the Solid State; Wiley: Chichester, 1999; pp 185-225.

(23) Melendez, R. E.; Hamilton, A. D. Hydrogen-Bonded Ribbons, Tapes and Sheets as Motifs for Crystal Engineering. In Top. Curr. Chem. 198, Design of Organic Solids; Weber, E., Ed.; Springer: Berlin, 1998; pp 97129.

(24) Copley, J. R. D.; Udovic, T. J. Neutron Time-of-Flight Spectroscopy. J. Res. Natl. Inst. Stand. Technol. 1993, 98, 71-97.

(25) Colognesi, D.; Celli, M.; Cilloco, F.; Newport, R. J.; Parker, S. F.; Rossi-Albertini, V.; Sacchetti, F.; Tomkinson, J.; Zoppi, M. TOSCA neutron spectrometer: the final configuration. Appl. Phys. A 2002, 74 (Suppl., Pt. 1), S64-S66.

(26) Hudson, B. S. Inelastic Neutron Scattering: A Tool in Molecular Spectroscopy and a Test of ab Initio Methods. J. Phys. Chem. A 2001, $105,3949-3953$

(27) Jaguar, v. 4.1; Schrodinger, Inc.: Portland, OR, 2000.

(28) Becke, A. D. Density-functional thermochemistry. III. The role of exact exchange. J. Chem. Phys. 1993, 98, 5648-5652.

(29) Stephens, P. J.; Devlin, F. J.; Chabalowski, C. F.; Frisch, M. J. Ab Initio Calculation of Vibrational Absorption and Circular Dichroism Spectra 
Using Density Functional Force Fields. J. Phys. Chem. 1994, 98, 1162311627.

(30) Hariharan, P. C.; Pople, J. A. Influence of polarization functions on MO hydrogenation energies. Theor. Chim. Acta 1973, 28, 213-22.

(31) Delley, B. An all-electron numerical method for solving the local density functional for polyatomic molecules. J. Chem. Phys. 1990, 92, 50817.

(32) Delley, B. Analytic energy derivatives in the numerical local -density functional approach. J. Chem. Phys. 1991, 94, 7245-50.

(33) Delley, B. From molecules to solids with the DMol ${ }^{3}$ approach. $J$. Chem. Phys. 2000, 113, 7756-7764.

(34) Wilson, E. B.; Decius, J. C., Jr.; Cross, P. C. Molecular Vibrations; Dover: New York, 1955; p 253. The "Wilson" numbering scheme for the normal modes of benzene is given here. A set of more recent values and a concordance with the more universal numbering scheme of Mulliken (and Herzberg) is given in L. D. Ziegler and B. Hudson, The Vibronic Spectroscopy of Benzene: Old Problems and New Techniques. In Excited States; Lim, E. C., Ed.; New York: Academic Press, 1982; Vol. 5, pp 41139.

(35) Jeffrey, G. A. An Introduction to Hydrogen Bonding; Oxford University Press: New York, Oxford, 1997; p 43.

(36) Emsley, J. Very Strong Hydrogen Bonding. J. Chem. Soc. Rev 1980, $91-124$.

(37) Speakman, J. C. Acid Salts of Carboxylic Acids. Crystals with Some 'Very Short' Hydrogen Bonds. Struct. Bonding 1972, 12, 141-199.

(38) Hadzi, D.; Orel, B.; Novak, A. Infrared and Raman spectra of some acid salts containing crystallographically symmetric hydrogen bonds. Spectrochim. Acta 1973, 29A, 1745-1753.

(39) Macdonald, A. L.; Robertson, J. M.; Speakman, J. C. A simple 'direct' solution of the crystallographica phase problem. Acta Crystallogr. 1971, B27, 2289-90.

(40) Guo, H.; Karplus, M. Solvent Influence on the Stability of the Peptide Hydrogen Bond: A Supramolecular Cooperative Effect. J. Phys. Chem. 1994, 98, 7104-5.
(41) Guo, H.; Karplus, M. Ab initio studies of hydrogen bonding of $\mathrm{N}$-methylacetamide: structure, cooperativity, and internal rotational barriers. J. Phys. Chem. 1992, 96, 7273-87.

(42) Kobko, N.; Dannenberg, J. J. Cooperativity in Amide Hydrogen Bonding Chains. Relation between Energy, Position, and $\mathrm{H}-$ Bond Chain Length in Peptide and Protein Folding Models. J. Phys. Chem. A 2003, 107, 10389-10395.

(43) Wieczorek, R.; Dannenberg, J. J. Hydrogen-Bond Cooperativity, Vibrational Coupling, and Dependence of Helix Stability on Changes in Amino Acid Sequence in Small 310-Helical Peptides. A Density Functional Theory Study. J. Am. Chem. Soc. 2003, 125, 14065-14071.

(44) Kobko, N.; Dannenberg, J. J. Cooperativity in Amide Hydrogen Bonding Chains. A Comparison between Vibrational Coupling through Hydrogen Bonds and Covalent Bonds. Implications for Peptide Vibrationa Spectra. J. Phys. Chem. A 2003, 107, 6688-6697.

(45) Dannenberg, J. J. Cooperativity in hydrogen bonded aggregates Models for crystals and peptides J. Mol. Struct. 2002, 615, 219-226.

(46) Kobko, N.; Paraskevas, L.; del Rio, E.; Dannenberg, J. J. Cooperativity in Amide Hydrogen Bonding Chains: Implications for ProteinFolding Models J. Am. Chem. Soc. 2001, 123, 4348-4349.

(47) Edison, A. S.; Weinhold, F.; Markley, J. L. Theoretical Studies of Protium/Deuterium Fractionation Factors and Cooperative Hydrogen Bonding in Peptides. J. Am. Chem. Soc. 1995, 117, 9619-24.

(48) Ludwig, R.; Reis, O; Winter, R.; Weinhold, F; Farrar, T. C. Quantum Cluster Equilibrium Theory of Liquids: Temperature Dependence of Hydrogen Bonding in Liquid $N$-Methylacetamide Studied by IR Spectra J. Phys. Chem. B 1998, 102, 9312-9318.

(49) Ludwig, R.; Weinhold, F.; Farrar, T. C. Experimental and theoretical studies of hydrogen bonding in neat, liquid formamide. J Chem. Phys. $\mathbf{1 9 9 5}$ $102,5118-25$

(50) Akiyama, M.; Torii, H. Cooperative effect in hydrogen bonding of $\mathrm{N}$-methylacetamide in carbon tetrachloride solution confirmed by NMR and IR spectroscopies. Spectrochim. Acta, Part A 2000, 56A, 137-144. 